
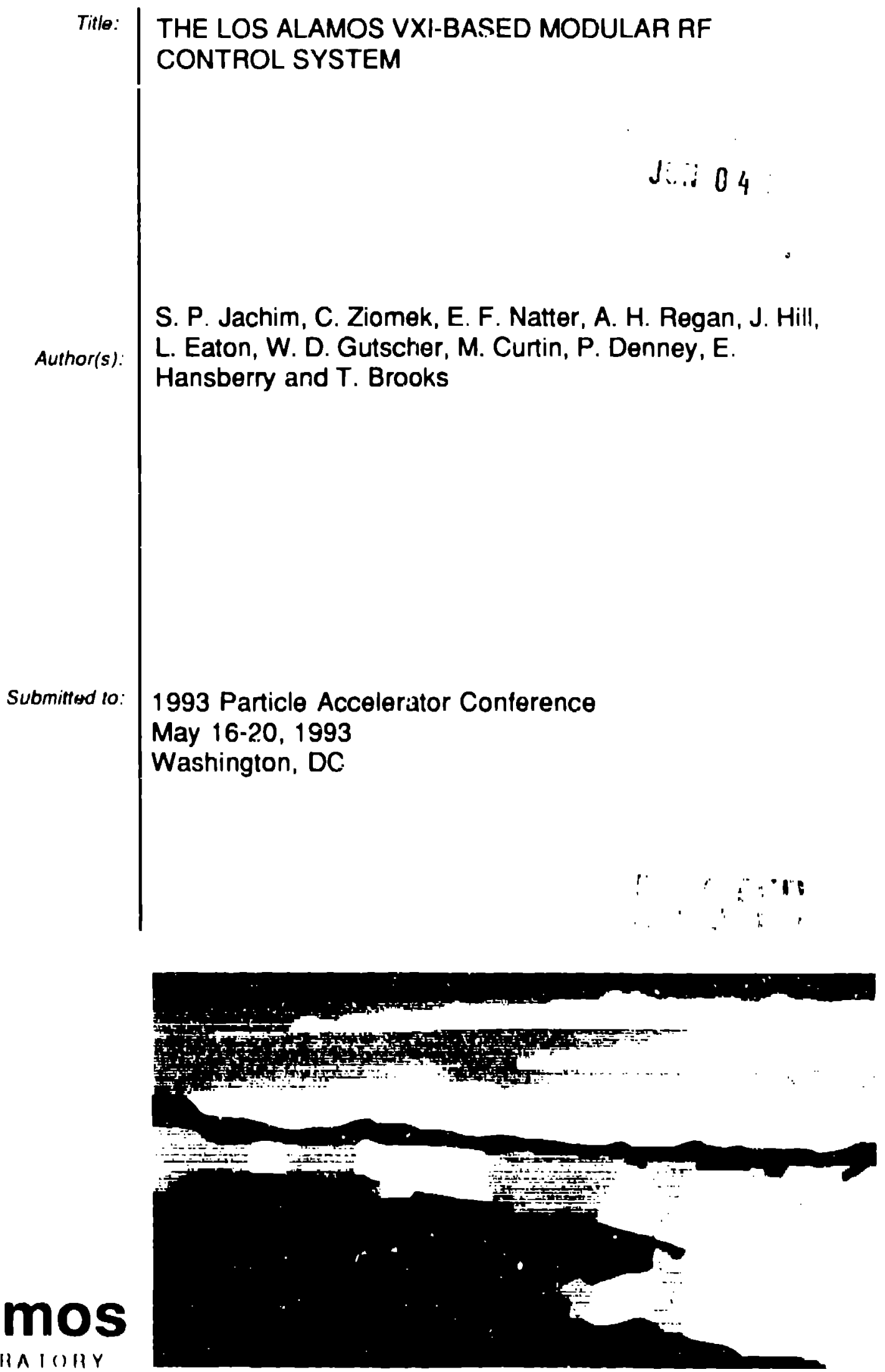

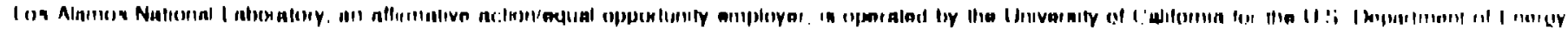

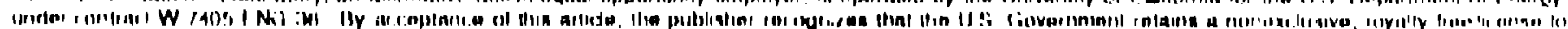

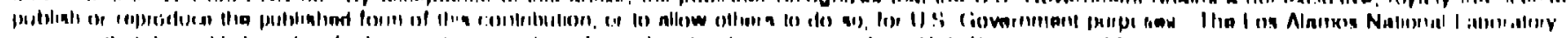

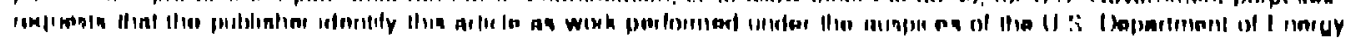




\title{
The Los Alamos VXI-Based Modular RF Control System*
}

\author{
S. P. Jachim, C. Zıomek, E. F. Natter, A. H. Regan. J. Hill, L. Faton, \\ W. D. Gutscher, M. Curtin, P. Denney, E. Hansberry, T. Brooks \\ MS-H827, Los Alamos National Laboratory, Los Alamos, NM 87545
}

\begin{abstract}
This paper desribes the design and implementation of the Ios Alamos modular RF control system, which provide, highnerformance teedhack and/or lecdforward control of RF accelerawr caviucs. This is a flexible, modular control sysicm which has been realized in the indusury-standard VXI cardmodular format. A wide spectrum of system functionality can be accommodated simply by incorporaung only uhose modules and features required for a particular application. The fundamental principles of the design approach are discussed. Detalls of the VXI implementation are given, including the system architecture and interfaces, pertormance capabilities, and availiable features.
\end{abstract}

\section{Introduction}

The AT-5 group at Los Alamos National Laboratory (LANL) is developing the RF system for the (iround Tesi Accelerator (GTA), including the RF control system, which is the topic of this paper. The GTA Program is a developmemt velaicle for Neutral Particle Beam (NPB) physics and technology

Because (iTA operates at several different harmonically. related trequencics using varicus power amplifier echnolugics, a decision was made rarly on to pursuc a moxtular RF control system architecture This architecture is designed w) be independent of RF: Irequency, power level, and type of accelerating structure. This approach has proven successiul on (iTA, and has allowed the same hartware to the used in a wade variety of other acceleratur applicaltons worlduide.

'Ihe lirst I.ANI. VXI moxlule for RI: conerol was (kesigned and huilt in log?. and the fourh complete control system was operated on (iTA in 190". Additionally, four other systems have heen installed and operalced at vartous other institutions. In all, alkout ('stac VXI molules luve he'"l busle to (batc.

\section{System Requircments}

The promery purpuse of an Rli comtrul system is lo lightly

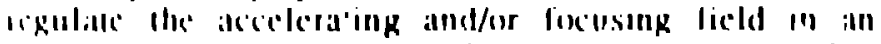

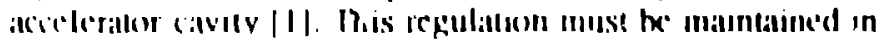

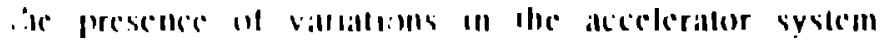

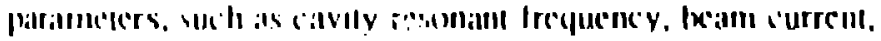

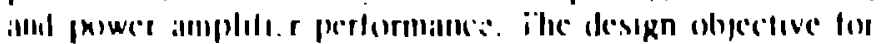

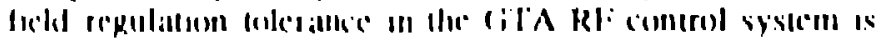

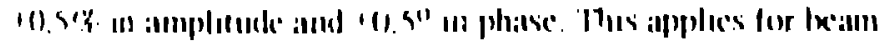
londony : (n)

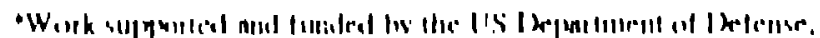

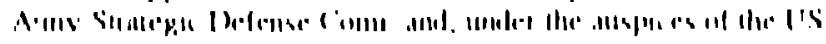

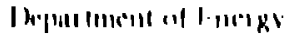

To achieve this level of regulation and to ensure elticient RF power uransier wo the cavily, the resonant frequency of thi accelerator cavity must be maintained within pressited limiss. A regulation tolerance of $\pm 2^{\circ}$ was chosen as an ohjective for the cavity reflectuon coeficicient phase on (jTA.

\section{System Architecture}

Figure 1 illustrates the essential aspects of the I.ANL RF control system architectu.e as applied (1) il particular acceleraung cavity $[2,3]$. A sample of the ciavily lichl is downconverted to a $20 \mathrm{MHz}$. IF and synchronously delected against the RF reference signal. The in-phase (I) illt yuadrature phise (Q) components of this detected signal aro compared to their respective commanded values, or w'(p)ints.

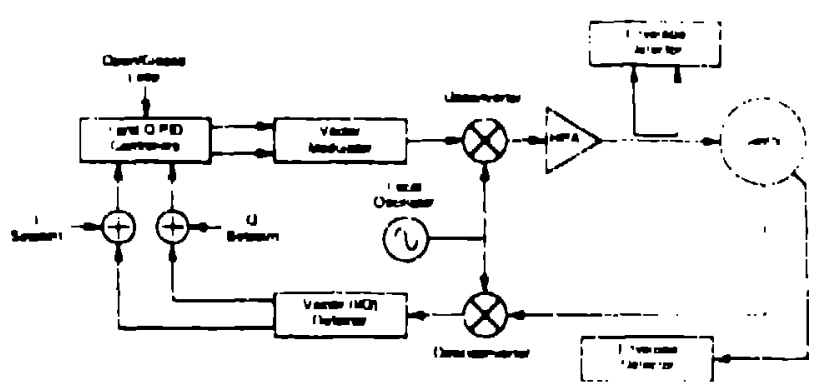

Figure 1, LANL RF control sysiem archite'clure'

The difference helween the detected compene'nts and the'is setpoints produces an emor vector. Ptopornional, mlegral, allul differential (PID)) closed-loxp control axtoons are then derived

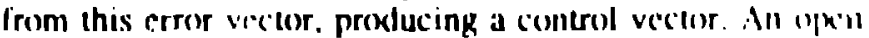
loop control vector ean optoonally he gencrated all the beginning of the acceleration cycle wo fill the (avily in at programmed mauner.

A vector modulator translates the control vis(ti) (1) it 2.1)MHz. IF carrer, which is subsequendy upcollvernid hill'k (1)

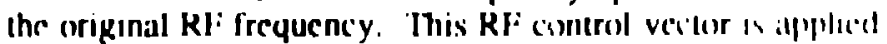

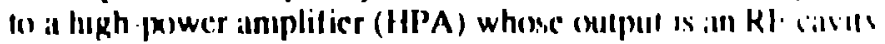
drive vecher, closing the fecellaick lixhy.

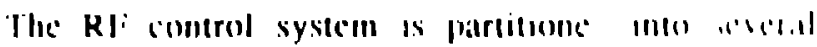
lunceional VXI modules as shown in tixure . The moklula,

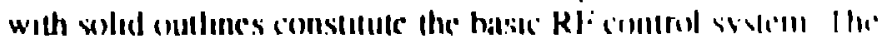

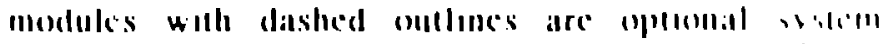

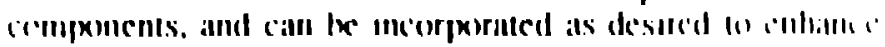

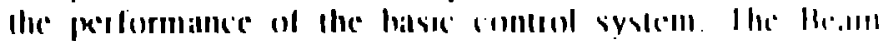

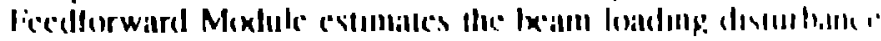

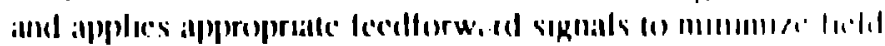

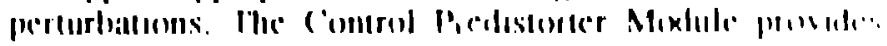

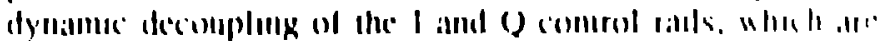

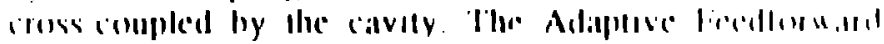

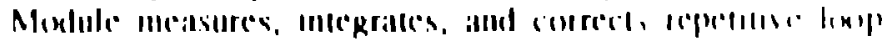

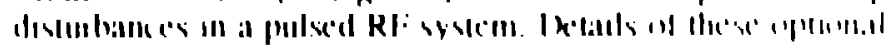

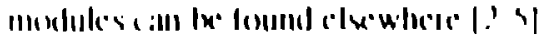




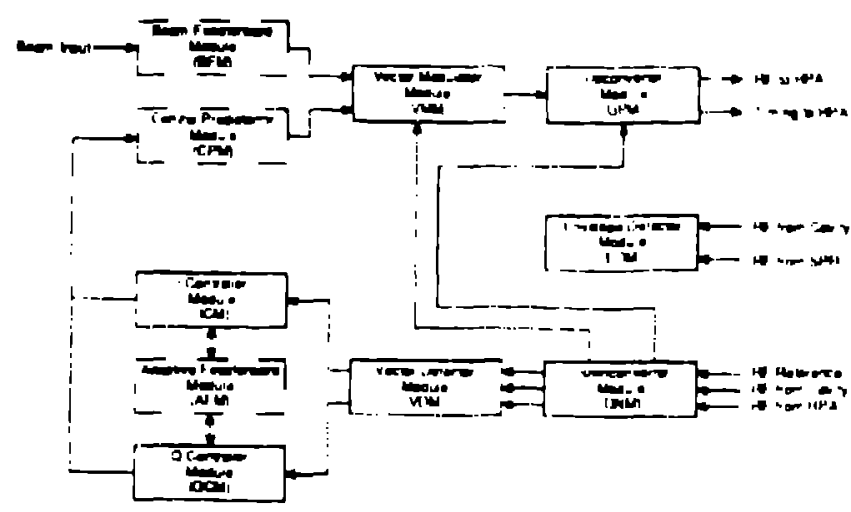

Figure 2. VXI moxlular implementation.

Feedback control is also employed to regulate the resonant frequency of the cavity [6.7]. As shown in ligure 3 . this is achieved by detecting the forward- and reverseuaveling waves in the cavity drive line, computing the cavity reflection coefficient, and taking corrective fecuback action by applying a control signal to a mechanical cavity tuner. Accuracy is assured by calibration and vector error correction [8]. All computations for this process are performed in software, and the feedback loop is closed through a virtual network connection to the tuner actualor.

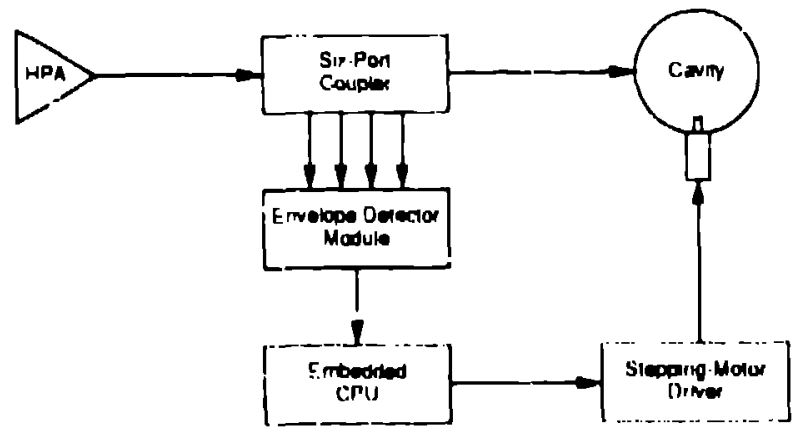

Figure 3. Resonance contul system.

Several ancillary functions are assigned to the RF control system as well. Measurements of various Rl: stgnal amplitudes in the system, such as the cavity ficld amplitude and (lrive, alle supported with Einvelope Ixecclor Modules. These moxlules sample and yuantize cach of eight RF inpue signials once per RI: puise.

The Rl: system assoxiated wilh each ravity is seyuenced and timed hy the RI control system. Thes lunclion is perlormed in the I!peonverter Module, whish sends ino

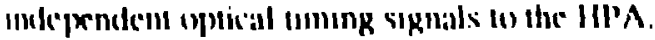

Ihe Rli system, like the rest of the ilcederalten, is required (1) operalce remolely under complete antomatum. Thens, supervisury compuler comerol, datal acepusslien, and remote

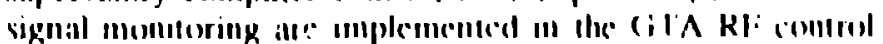

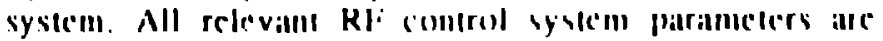

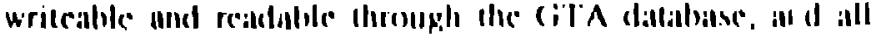

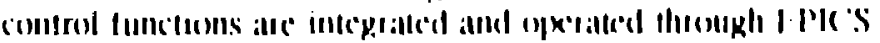
cimirul screns.

\section{Hardware Realization}

The VME Extensions for Instrumentiluon ( V Xl) slundtar! 110) which energed in the 1988-84 time frame was develupes! by a consortium of leading instrument manutacturers. This standard builds on, and is compatible with. the ubiquilous anit powerful VME standard to produce a robust card-mixlular medium capable of supporting high-performance undiog. digital and microwave instruments. The design uf lice RF control system commenced in this ume period, and VXI His chosen as the packaging medium. VXI held promise of not only supporing the needs of the RF contol hardwise, but al wo of litung seamlessly into the GTA compuk'r control syite'm, it being of a V'ME-based distributed archilecture.

The promise of VXI has held une in practice. Most of thi if control modules have been, of necessily, designed in house. In all cases, strict adherence to the VXI standard his been mantained. Several commercial VMF cuards, such dis al 680)20-bused processor and an Ethernet interface card, hall already been integrated into the computer contur system. These VME modules were directly embedded in the VXI RI. conurol system and integrated together withoul itgnilicillt difficulty.

Figure 4 shows a conceptual layout of a lypial l.A.Yl VXI module. Details of the register-based VXI Interlit:l' can be found elsewhere [9]. All timing and signal comversion functions are distributed down to the module level. $A$ II).111, clock and a synchronizing urigger are broadeast will mixluh on the VXI backplane. Counters on each module's intertitis circuit count down from preloaded register values anti provide on hoard timing signals, As needrd, AVD and 1$) / A$ (anverters arc provided in situ on each module. Signal simplans: is tripgered by the on-board timing signals, which are under soliware control. 'This approach greatly simplilics istem integration and configuration managememe. Becaluse the number of connectors and cahles on the system has heoll minimized, reliability is improved.

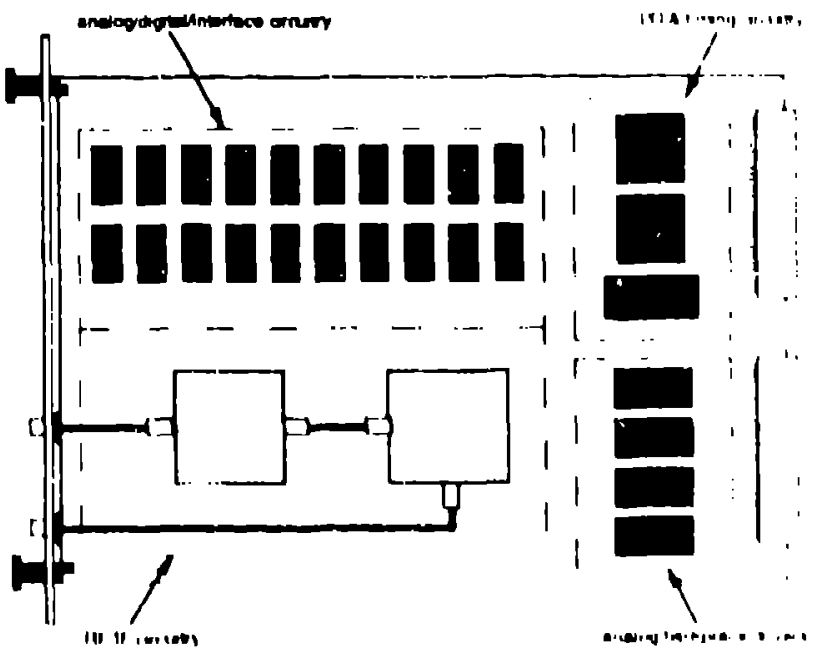

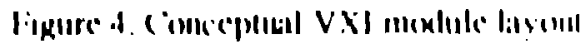

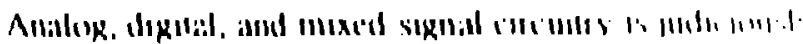

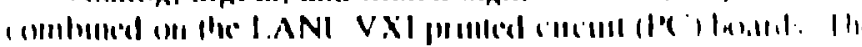

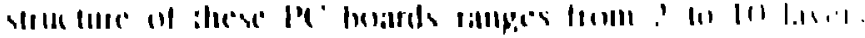




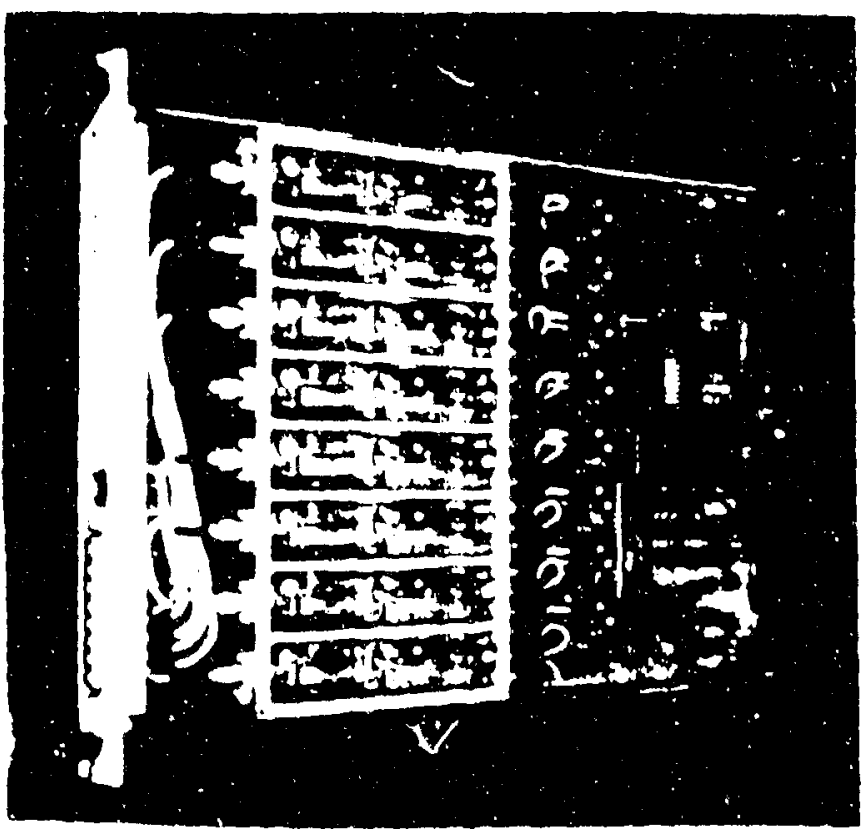

$k, 1,1,1]$, 


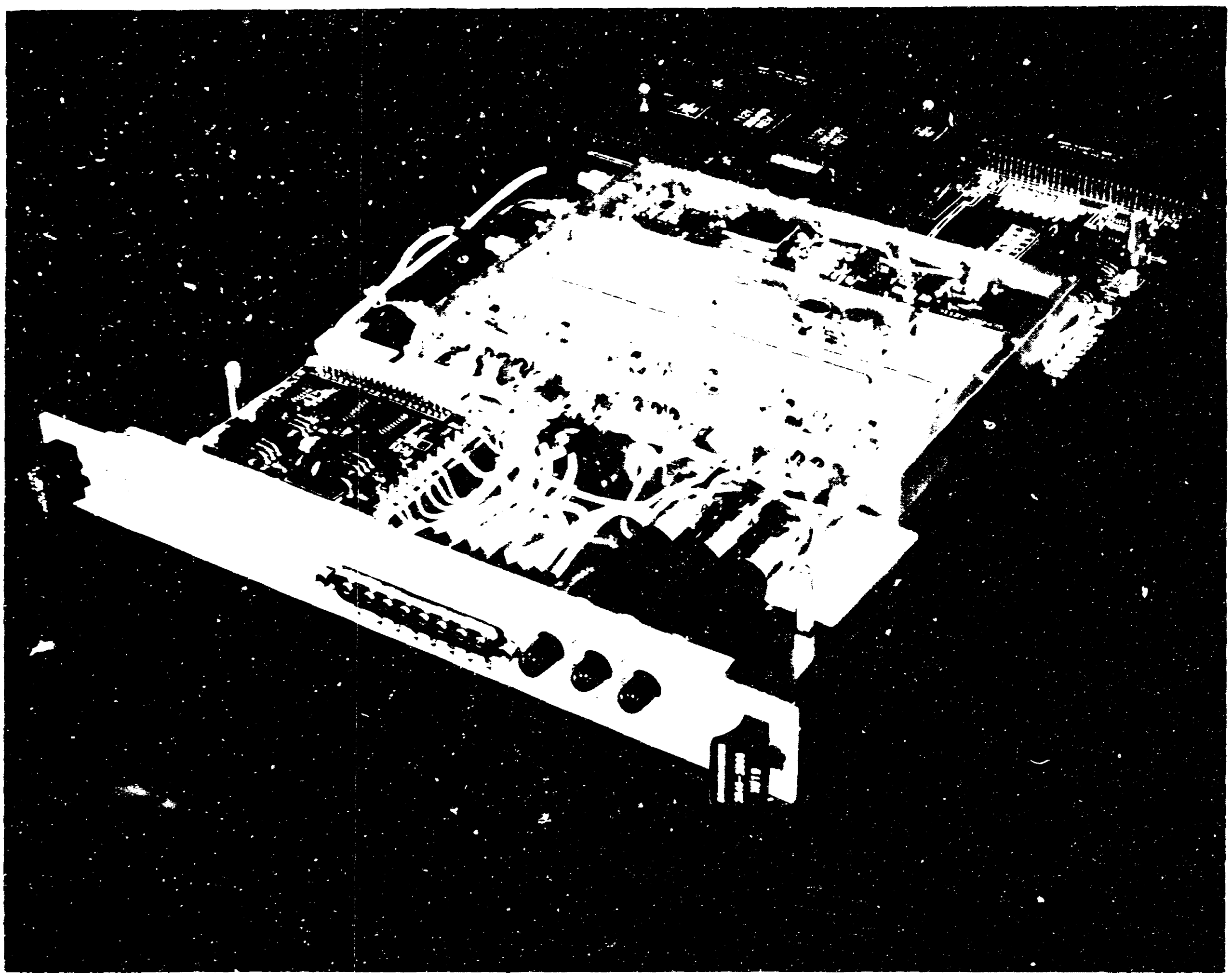




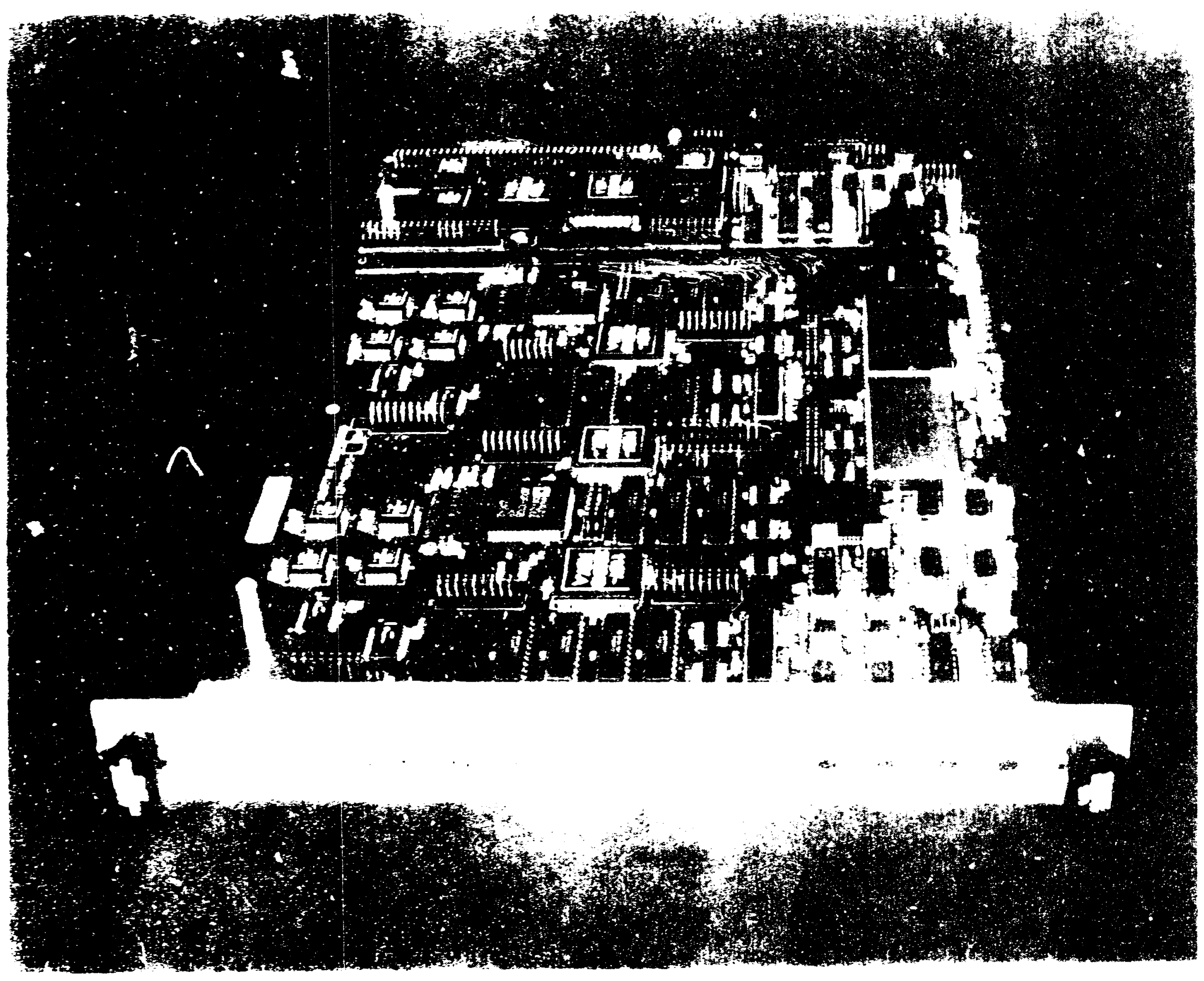

